\title{
Green synthesis and characterization of gold nanoparticles using leaf extracts of Withania somnifera (Linn.) (Ashwagandha)
}

\author{
Birendra Kumar Bindhani, ${ }^{1, ~ *}$, Ashok Kumar Panigrahi ${ }^{2}$ \\ ${ }^{1}$ School of Biotechnology, KIIT University, Bhubaneswar, Odisha, India \\ ${ }^{2}$ Department of Botany, Berhampur University, Odisha, India
}

Email address:

drbindhani@gmail.com (B. K. Bindhani)

To cite this article:

Birendra Kumar Bindhani, Ashok Kumar Panigrahi. Green synthesis and Characterization of Gold Nanoparticles Using Leaf Extracts of Withania somnifera (Linn.) (Ashwagandha). International Journal of Materials Science and Applications. Vol. 3, No. 6, 2014 , pp. $279-284$. doi: 10.11648/j.ijmsa.20140306.11

\begin{abstract}
In this study, a novel technique for biosynthesizing of gold nanoparticles (GNPs) using Withania somnifera as reductants and stabilizers is reported. Biosynthesis of anisotropic gold nanoparticles using ethanolic extract of Withania somnifera leaf and their potential as IR blockers has been demonstrated. The phenolic groups $(-\mathrm{OH})$ residue was identified as the active functional group for gold ion reduction. These gold nanoparticles were characterized by of UV-Vis spectrophotometer, FTIR, TEM. The presence of proteins was identified by FTIR, UV-Vis and fluorescence spectroscopy. The micrograph revealed the formation of anisotropic gold nanoaprticles. The characteristics of the nanoparticles formed suggest application of gold and gold nanoparticles as chemical sensors in the future. Given the simple and eco-friendly approach for synthesis, these nanoparticles could easily be commercialized for large scale production. The present research opens a new avenue for the green synthesis of nanomaterials.
\end{abstract}

Keywords: Green Synthesis, Withania Somnifera, Ethanolic Extract, Gold Nanoparticles

\section{Introduction}

Nanotechnology is an important field of modern research dealing with design, synthesis, and manipulation of particles structure ranging from approximately $1-100 \mathrm{~nm}$. Tremendous growth in this emerging technology has opened novel fundamental and applied frontiers, including the synthesis of nanoscale materials and exploration or utilization of their exotic physicochemical and optoelectronic properties. Nanotechnology is rapidly gaining importance in a number of areas such as health care, cosmetics, food and feed, environmental health, mechanics, optics, biomedical sciences, chemical industries, electronics, space industries, drug-gene delivery, energy science, optoelectronics, catalysis, reprography, single electron transistors, light emitters, nonlinear optical devices, and photo electrochemical applications [1-9]. Gold nanoparticles are of interest because of the unique properties (e.g., size and shape depending optical, electrical, and magnetic properties) which can be incorporated into antimicrobial applications, biosensor materials, composite fibres, cryogenic superconducting materials, cosmetic products and electronic components. Several physical and chemical methods have been used for synthesizing and stabilizing gold nanoparticles [9-11]. The most popular chemical approaches, including chemical reduction using a variety of organic and inorganic reducing agents, electrochemical techniques, physicochemical reduction and radiolysis are widely used for the synthesis of gold nanoparticles. Recently, nanoparticle synthesis is among the most interesting scientific areas of inquiry and there is growing attention to produce nanoparticles using environmentally friendly methods (green chemistry).

Green synthesis approaches include mixed-valence polyoxometalates, polysaccharides, Tollens, biological and irradiation method which have advantages over conventional methods involving chemical agents associated with environmental toxicity. This article presents an overview of gold nanoparticle preparation by green synthesis approaches. Therefore, to reflect on the current state and future prospects, especially the potentials and limitations of the above mentioned techniques for industries [12]. 
Withania somnifera, is an erect, evergreen, perennial shrub which is a widely used medicinal plant considered as aphrodisiac and rejuvenating, anti-inflammatory and anti tumor agent [13]. It is widely used as an important drug in Ayurvedic formulations. The genus Withania somnifera belongs to the division Magnoliophyta, class Magnoliopsida, order Solanales and family Solanaceae [14-20]. It is best known classically for its rejuvenating properties and hence called "Indian Ginseng" [21-25]. Roots of Withania somnifera (Ashwagandha) reportedly exhibit antioxidant, immune modulatory and haematopoietic properties [26-30]. Phytochemicals (from the Greek word phyto, meaning plant) are biologically active, naturally occurring chemical compounds found in plants, which provide health benefits for humans than those attributed to macronutrients and micronutrients [30-32].

In present study anisotropic gold nanoparticles were synthesized using Withania somnifera leaf ethanolic extract and demonstrated their potential in absorption of infrared rays. These green synthesized nanoparticles were examined by ultraviolet visible spectroscopy, transmission electron microscopy (TEM), energy dispersive X-ray analysis (EDAX), and Fourier transform infrared (FTIR) spectroscopy to determine their size and shape.

\section{Materials and Methods}

\subsection{Plant Material Source}

Withania somnifera leaf (Figure 1a) was procured from the local market and planted in earthen pots at Green house of Botanical Garden, Department of Botany, Berhampur University, Odisha, India.

\subsection{Preparation of Crude Extracts}

The air dried leaf and stem were milled to get a coarse powder. About $100 \mathrm{~g}$ of dry powder was extracted with petroleum ether at room temperature using soxhlet apparatus for $8 \mathrm{hrs}$ or the extraction was continued until the liquid was clear (Figure 1b). The extracts were then filtered and concentrated to a dry mass under vacuum. The mark left after petroleum ether extraction was air dried and then extracted with solvents ethanol as done earlier and the extracts were similarly filtered and concentrated under vacuum.

\subsection{Qualitative Chemical Evaluation}

The different extracts thus obtained were qualitatively tested for the presence of various phytochemical constituents (Table 1) [26-30].

\subsection{Synthesis of Gold Nanoparticles}

$100 \mathrm{ml}$ of plant or leaf extract were collected in a $500 \mathrm{ml}$ beaker which was kept on magnetic stirrer for stirring at 200 rpm at room temperature (Figure 1c), to this stirring solution $10 \mathrm{ml}$ of $1 \mathrm{mM}$ auric acid solution was added drop wise. The ratio of extract and auric acid solution in above procedure is
10:1, similarly $5: 1$ and $1: 1$ ratios were followed for optimization of protocol [33] (Figure 1d).
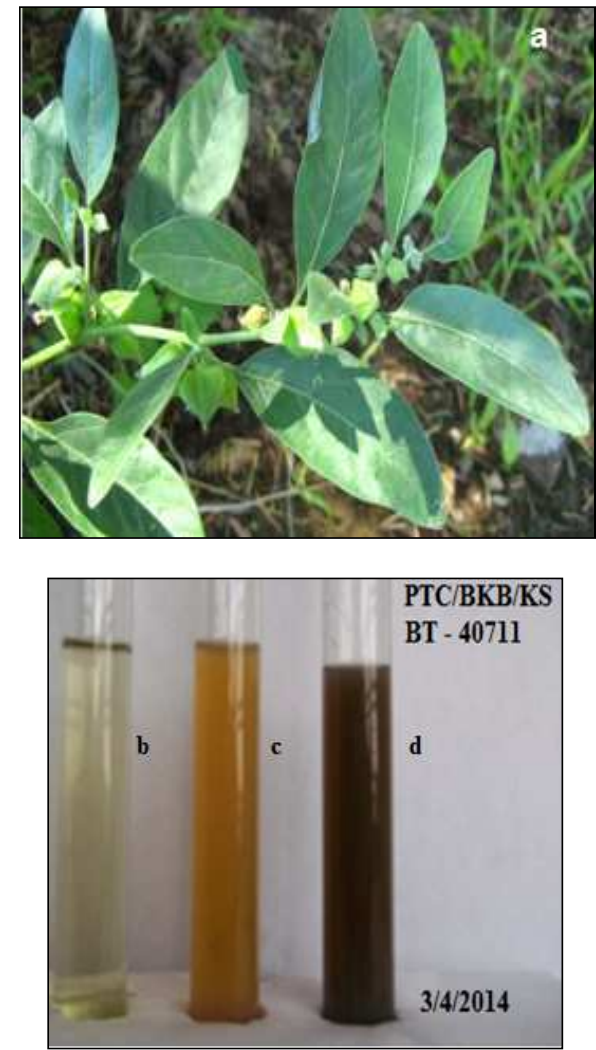

Figure 1. Withania somnifera leaf (a), ImM aqueous $\mathrm{HAuCl}_{4}$ solution (b) leaf Extract (c) and AuNPs (d)

Table 1. Preliminary phytochemically analysis of various extract of Withania somnifera leaf extract.

\begin{tabular}{lll}
\hline Sl. No. & Phytochemical Constituents & Ethanol Extraction \\
\hline 1 & Flavonoids & +++ \\
2 & Alkaloids & +++ \\
3 & Steroids & ++ \\
4 & Phenols & +++ \\
5 & Carboxylic Acids & +++ \\
6 & Xantho Proteins & ++ \\
7 & Terpenoids & +++ \\
8 & Quinones & ++ \\
\hline
\end{tabular}

$(++)=$ moderately present,$(+++)=$ Appreciable amount

\section{Measurements}

\subsection{UV-Vis Spectroscopy}

Ultraviolet-visible spectroscopy (UV-1601 pc shimadzu spectrophotometer) or ultraviolet-visible spectrophotometer (UV-Vis) refers to absorption spectroscopy in the UV-Visible spectral region.

\subsection{Fourier Transmission Infra Red Spectroscopy (FTIR)}

The FTIR spectrum of Withania somnifera L. extract, gold nanoparticles and amine functionalized gold nanoparticles were recorded using were obtained using a BIORAD-FTS7PC type FTIR spectrophotometer. 


\subsection{EDAX Spectroscopy}

Energy-dispersive $\mathrm{X}$-ray spectroscopes exploit the photon nature of light. In the X-ray range, the energy of a single photon is just sufficient to produce a measurable X-ray voltage pulse, and the output of an ultralow noise preamplifier connected to the low noise constitutes a statistical measure of the corresponding quantum energy. By digitally recording and counting a large number of such pulses within a so-called multichannel analyzer, a complete image of the X-ray spectrum is built up almost simultaneously.

\subsection{Transmission Electron Microscope (TEM) Analysis}

Transmission electron microscope (TEM) (Philips CM-10) is a microscopy technique whereby a beam of electrons is transmitted through an ultra-thin specimen, interacting with the specimen as it passes through. An image was formed from the interaction of the electrons transmitted through the specimen; the image was magnified and focused onto an imaging device.

\subsection{Statistical Analysis}

All values are expressed as means \pm standard deviation. The results were analyzed using one-way analysis of variance (ANOVA), and the differences among the formulation means were analysed using the Tukey-Kramer multiple-comparison test. The software Graph Pad in Stat was employed for the statistical analysis.

\section{Results and Discussions}

\subsection{UV-Vis Spectrophotometry}

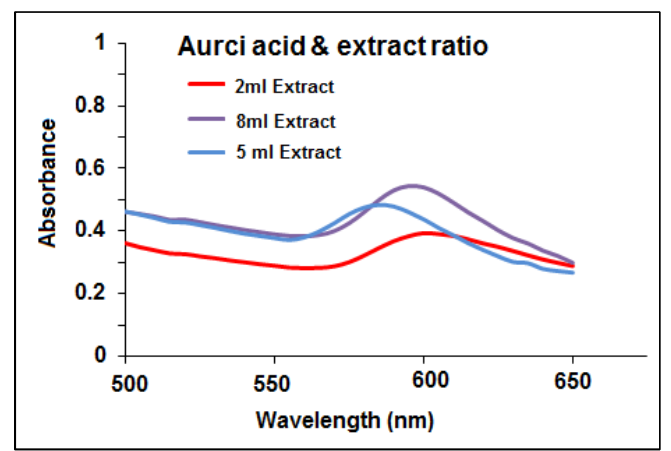

Figure 2. UV-Visible absorption spectrum of gold nanoparticles of Withania somnifera L. leaf extract.

Gold nanoparticles were characterized by UV visible spectrophotometer. Figure 2 shows the GNP formation using a constant $\mathrm{HAuCl}_{4}$ concentration $\left(1.3 \times 10^{4} \mathrm{M}\right)$ with different concentrations of the extract. Photographs of figure-1 shows the color change of the gold nanoparticles with a changing Withania somnifera leaf extract concentration. The violet, pink to dark pink color observed is characteristic for the Surface Plasmon Resonance (SPR) of different sizes of gold nanoparticles [34]. The leaf extract quantities were varied from
$2 \mathrm{ml}$ to $10 \mathrm{ml}$, particularly in the range of low amounts of the leaf extract $(2-5 \mathrm{ml}$ in $10 \mathrm{ml}$ metal ion solution), the absorption spectra exhibit a gradual increase of the absorbance accompanied with a shift in the $\mathrm{k}_{\max }$ from 545 to $530 \mathrm{~nm}$.

A strong correlation between the particle size and the maximum absorption peak has been previously observed [35]. They observed that as the particle size increases, the maximum absorption is red shifted. Accordingly, it can be concluded that the size of the particles decreases upon increasing the extract concentration from 0.2 to $2 \mathrm{ml}$ as the absorption spectra are blue shifted [33]. The slight red shift in $\lambda_{\max }$ accompanied with a slight increase in the absorbance values at longer wavelengths $(600 \mathrm{~nm})$ with an increase in the extract concentration from 3 to $6 \mathrm{ml}$ is consistent with previously reported results for surfactant [34].

\subsection{FTIR}

The nature of amino acid residue in the plant protein responsible for gold ion reduction revealed by FTIR and fluorescence analysis of protein structure before and after reaction with metal ions is shown in Figure 3. The FTIR spectrum (Figure 3a) showed the bands at the interaction of nanoparticles with phytochemicals of Withania somnifera showed intense peaks at 2883,1602, 1504, 1383, 1071, and $1333 \mathrm{~cm}^{-1}$ relative shift in position and intensity distribution were confirmed with FTIR. The FTIR recorded (Figure 3b) for the dry Withania somnifera powder showed strong bands at $2831,1733,1611$, and $1402 \mathrm{~cm}^{-1}$. Comparing the FTIR spectra, it can be seen that for changes in the $-\mathrm{COOH}$ group for $-\mathrm{OH}$, i.e., the hydroxyl group, the peak appeared at $3314 \mathrm{~cm}^{-1}$ in the raw material, but after encapsulation of nanoparticles, the peak was narrower and shifted to $3633 \mathrm{~cm}^{-1}$ and the peak intensity reduced also for the $-\mathrm{C}-$ of the carboxylic group after encapsulation of nanoparticles. H bonds can be formed between the amide groups. This implies that the $-\mathrm{COOH}$ group in the compound was attached to the gold nanoparticles and there is a clear change in the spectra. After encapsulation, the $1732 \mathrm{~cm}^{-1}$ in $-\mathrm{C}$ - bond stretching becomes masked or disappears $[35,36]$.

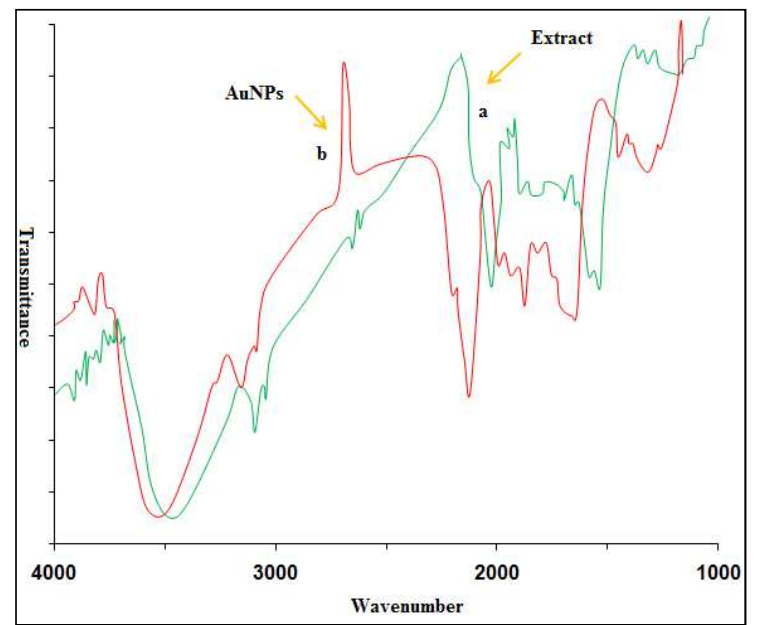

Figure 3. FTIR spectrum of plant protein from the plant extract (a) and reaction with gold ions (b). 


\section{3. $E D A X$}

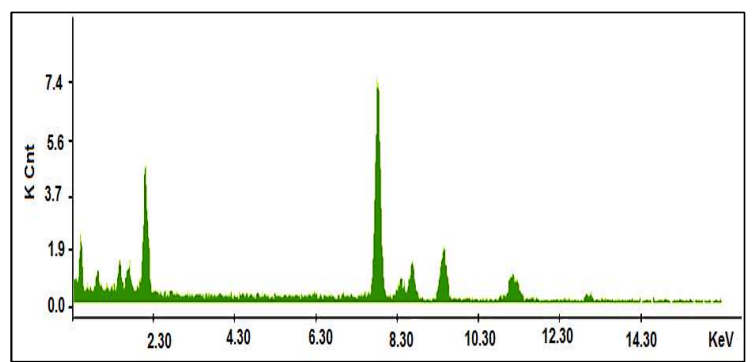

Figure 4. EDAX profile of synthesized Gold nanoparticles.

The green synthesized gold nanoparticles were investigated further using EDAX spectrometry, which confirmed the presence of gold with no contaminants. The optical adsorption peak was observed at approximately 2.30 $\mathrm{keV}$ (Figure 4), which is typical of adsorption of gold nanocrystallites due to surface plasmon resonance. The EDAX profile for gold nanoparticles synthesized using Withania somnifera showed strong gold atom signals at around 2.30, 8.10, 9.40, and $11.30 \mathrm{keV}$. EDAX for the gold nanoparticles showed strong signals for gold atoms and weaker signals for carbon, oxygen, and chloride, which were provenient from biomolecules of Withania somnifera, and the strong signals obtained at around $2.30 \mathrm{keV}$ and $9 \mathrm{keV}$ were similar to those in our earlier studies [37, 38].

\subsection{TEM}

This result was confirmed by TEM measurements at $\mathrm{pH}$ 3.5 and 9.6 (Figure 5). The sizes of the particles at $\mathrm{pH} 3.5$ and pH9.6 were larger while forming hexagonal structures. Furthermore, the particles formed in acidic medium were unstable and precipitated within $12 \mathrm{~h}$ [39-46].

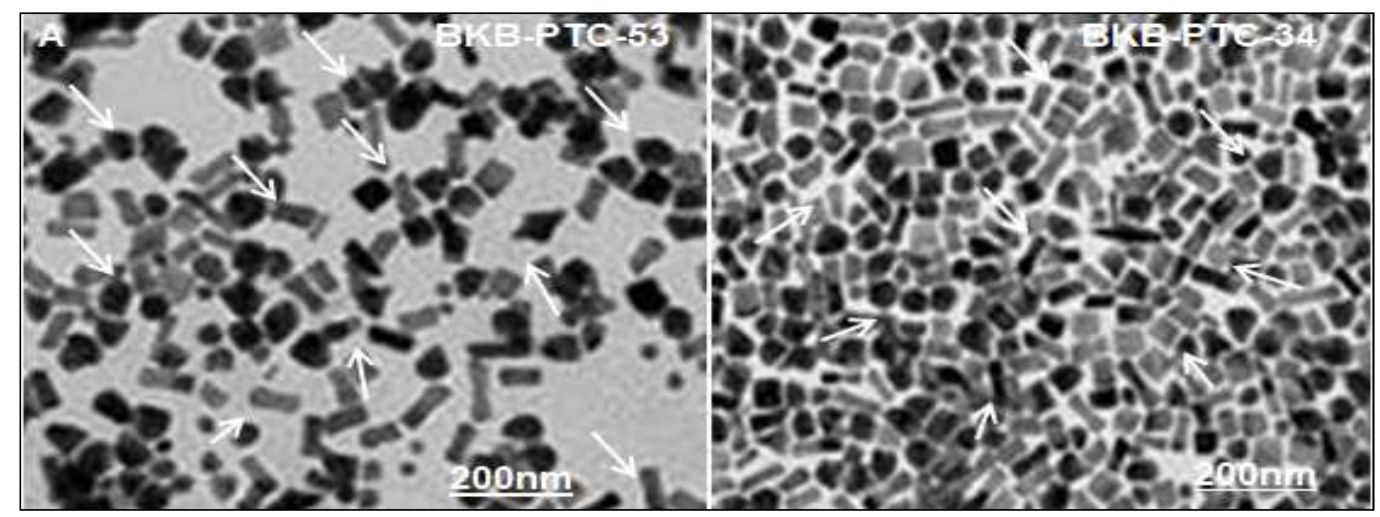

Figure 5. TEM images of AuNPs at (A) pH 3.5 and (B) pH 9.6.

\section{Conclusion}

The present study demonstrates bioreductive synthesis of nanosized gold particles using Withania somnifera ethanol leaf extract appears to be environmentally friendly, so that this protocol could be used for rapid production of gold nanoparticles. The size of the nanoparticles can be easily adjusted by using different amounts of leaf extract. The high phenolic content of the hot water extract of olive leaves having strong anti-oxidant properties helped in the reduction of gold cations to AuNPs. The characterization of AuNPs revealed that the morphology of the AuNPs depends on the extract concentration and $\mathrm{pH}$ of the used medium. At higher concentration of the extract and basic $\mathrm{pH}$, the pseudospherical particles are capped by phytochemicals. In the future, selection of such plants may create a new platform for realizing the potential of herbal medicines in nanoscience for drug delivery and biomedical application.

\section{Acknowledgement}

We are deeply thankful to Director, School of Biotechnology, KIIT University, Bhubaneswar, Odisha, India and Department of Botany, Berhampur University, Odisha, India for their valuable help during this investigation.

\section{References}

[1] Berciaud S, Cognet L, Tamarat P, Lounis B. Observation of intrinsic size effects in the optical response of individual gold nanoparticles. Nano Letters. 2005; 5(3): 515-518.

[2] Bhainsa KC, D'Souza SF. Extracellular biosynthesis of silver nanoparticles using the fungus Aspergillus fumigates. Colloids and Surfaces B: Biointerfaces. 2006; 47: 160- 164.

[3] Bhol KC, Schechter PJ. Effects of Nanocrystalline Silver in a Rat Model of Ulcerative Colitis. Digestive Science. 2007; 52: $2732-2742$.

[4] Bogle KA, Dhole SD, Bhoraskar VN. Silver nanoparticles: synthesis and size control by electron irradiation. Nanotechnology. 2006; 17: 3204 - 3208.

[5] Bogunia-Kubik K, Sugisaka M. From molecular biology to nanotechnology and nanomedicine. Biosystems. 2002; 65(25):123-138

[6] Boyd DA, Greengard L, Brongersma M, El-Naggar MY, Goodwin D. Plasmon-Assisted Chemical Vapor Deposition. Nano Letters. 2006; 6 (11): 2592 - 2597.

[7] Braydich-Stolle L, Hussain S, Schlager JJ, Hofmann MC. In Vitro Cytotoxicity of Nanoparticles in Mammalian Germline Stem Cells. Toxicological Sciences. 2005; 88 (2):.412 - 419. 
[8] Brust M, Kiely C. Some recent advances in nanostructure preparation from gold and silver particles: a short topical review. Colloids and Surfaces A: Physiochemical and Engineering Aspects. 2002; 202(2-3):175 - 186.

[9] Cao G. Nanostructures and nanomaterials: synthesis, properties and applications. Imperial College Press. 2004; ISBN: 1-86094-4159, London

[10] Chang ALS, Khosravi V, Egbert B. A case of argyria after colloidal silver ingestion. Journal of Cutaneous Pathology. 2006; 33 (12): $809-811$.

[11] Chen J, Wang J, Zhang X, Jin Y. Microwave-assisted green synthesis of silver nanoparticles by carboxymethyl cellulose sodium and silver nitrate. Materials Chemistry and Physics. 2008; 108: $421-424$.

[12] Smitha SL, Philip D, Gopchandran KG. Green synthesis of gold nanoparticles using Cinnamomum zeylanicum leaf broth. Spectrochim Acta A Mol Biomol Spectrosc. 2009; 74(3):735 739.

[13] Chen W, Cai W, Zhang L, Wang G, Zhang L. Sonochemical Processes and Formation of Gold Nanoparticles within Pores of Mesoporous Silica. Journal of Colloid and Interface Science. 2001; 238 (2): 291 - 295.

[14] Costa MA, Zia ZQ, Davin LB, Lewis NG. 1999. Chapter Four: Toward Engineering the Metabolic Pathways of CancerPreventing Lignans in Cereal Grains and Other Crops. In Recent Advances in Phytochemistry, vol. 33, Phytochemicals in Human Health Protection, Nutrition, and Plant Defense, ed. JT Romeo, New York, 67-87.

[15] Devmurari VP, Jivani NP. Phytochemical Screening and Antibacterial Activity of Ethanolic Extract of Artemisia nilagirica. Annals of Biological Research. 2010; 1 (1), 10-14.

[16] Elsakka M, Grigorescu E, Stanescu U, Stanescu U, Dorneanu V. New data referring to chemistry of Withania somnifera species. Rev Med Chir Soc Med Nat Iasi. 1990; 94: 385 - 387.

[17] Grierson DS, Afolayan AJ. Antibacterial activity of some indigenous plants used for the treatment of wound in the Eastern Cape, S. Afr. J. Ethnopharmacol. 1999; 66: 103 -106.

[18] Gibson EL, Wardel J, Watts CJ. Fruit and Vegetable Consumption, Nutritional Knowledge and Beliefs in Mothers and Children. Appetite. 1998; 31: 205-228.

[19] Heiser CB, Smith PC. The cultivated Capsicum. Econ. Bot. $1953 ; 214-227$.

[20] Harborne JB. Phytochemical Methods: A Guide to Modern Techniques of Plant Analysis, Springer Netherlands, 1984.

[21] Harborne JB, Baxter H. Phytochemical dictionary: a handbook of bioactive compounds from plants. 4 John St. London: Taylor \& Francis Ltd. 1995.

[22] Harborne JB. Phytochemical methods (2nd Ed). Chapman and Hall, London and New York. 1984; Pp 288.

[23] Hasler CM, Blumberg JB. Symposium on Phytochemicals: Biochemistry and Physiology. Journal of Nutrition. 1999; 129: 756S-757S.

[24] Kupchan SM, Doskotch RW, Bollinger P, Muphail AT, Sim GA, Saenz RJ. The isolation and structure elucidation of a novel steroidal tumor inhibitor from Acnistus arborescens. J. Am. Chem. Soc. 1965; 87:5805.
[25] Lingaiah M, Nagaraja Rao P. An ethnobotanical survey of medicinal plants used by traditional healers of Adilabad district, Andhra Pradesh, India. Biolife. 2013; 1(1):17-23.

[26] Mathai K. Nutrition in the Adult Years. In Krause's Food, Nutrition, and Diet Therapy, 10th ed., ed. L.K. Mahan and S. Escott-Stump, American Cancer Society. 2000; 271: 274-275.

[27] Mishra LC, Singh BB, Dagenais S. Scientificbasis for the therapeutic use of Withania somnifera (Ashwagandha), a review. Altern. Med. Rev. 2000; 5: 334 - 346.

[28] Naidu PS, Singh A, Kulkarni SK. Effect of Withania somnifera root extract on haloperidol induced orofacial dyskinesia: Possible mechanism of action. J. Med. Food, 2003; 6: $107-114$.

[29] Rajeshwar Y, Lalitha R. Preliminary phytochemical screening and in vitro anthelmintic effects of Acmella paniculata plant extracts. Biolife. 2013; 1(3):106 -112.

[30] Singh S, Kumar S, Withania somnifera - The Indian Ginseng Ashwagandha. Central Institute of Medicinal and Aromatic Plants, Lucknow, India, 1998.

[31] Sharma S, Sharma MC, Kohli DV. In vitro micro propagation of medicinally important roots and axillary bud combination. Journal of Optoelectronic and biomedical materials. 2009; 4: $379-381$.

[32] Sofowora A. Medicinal Plants and Traditional Medicine in Africa. 2nd ed., Spectrum Books Ltd., Ibadan, Nigeria. 1993.

[33] Treas, GE, Evans, "Pharmacognocy", English language Book Society, Bailliere Tindall, London. 1985; pp: 394.

[34] Arunachalam KD, Annamalai SK. Chrysopogon zizanioides aqueous extract mediated synthesis, characterization of crystalline silver and gold nanoparticles for biomedical applications. Int J Nanomedicine. 2013; 8:2375-84.

[35] Arunachalam KD, Annamalai SK, Hari S. One-step green synthesis and characterization of leaf extract-mediated biocompatible silver and gold nanoparticles from Memecylon umbellatum. Int J Nanomedicine. 2013; 8:1307-15.

[36] Annamalai A, Christina VL, Sudha D, Kalpana M, Lakshmi PT. Green synthesis, characterization and antimicrobial activity of Au NPs using Euphorbia hirta L. leaf extract. Colloids Surf B Biointerfaces. 2013; 108:60-5.

[37] Dubey SP, Dwivedi AD, Lahtinen M, Lee C, Kwon YN, Sillanpaa M. Protocol for development of various plants leaves extract in single-pot synthesis of metal nanoparticles. Spectrochim Acta A Mol Biomol Spectrosc. 2013; 103:134-42.

[38] Tamuly C, Hazarika M, Borah SCh, Das MR, Boruah MP. In situ biosynthesis of $\mathrm{Ag}, \mathrm{Au}$ and bimetallic nanoparticles using Piper pedicellatum C.DC: green chemistry approach. Colloids Surf B Biointerfaces. 2013; 102:627-34.

[39] Mukherjee S, Sushma V, Patra S, Barui AK, Bhadra MP, Sreedhar B, Patra CR. Green chemistry approach for the synthesis and stabilization of biocompatible gold nanoparticles and their potential applications in cancer therapy. Nanotechnology. 2012; 23(45):455103.

[40] Noruzi M, Zare D, Davoodi D. A rapid biosynthesis route for the preparation of gold nanoparticles by aqueous extract of cypress leaves at room temperature. Spectrochim Acta A Mol Biomol Spectrosc. 2012; 94: 84 - 88. 
[41] Elavazhagan T, Arunachalam KD. Memecylon edule leaf extract mediated green synthesis of silver and gold nanoparticles. Int J Nanomedicine. 2011; 6:1265 - 1278.

[42] Kumar VG, Gokavarapu SD, Rajeswari A, Dhas TS, Karthick V, Kapadia Z, Shrestha T, Barathy IA, Roy A, Sinha S. Facile green synthesis of gold nanoparticles using leaf extract of antidiabetic potent Cassia auriculata. Colloids Surf B Biointerfaces. 2011; 87(1):159 - 163.

[43] Noruzi M, Zare D, Khoshnevisan K, Davoodi D Rapid green synthesis of gold nanoparticles using Rosa hybrida petal extract at room temperature. Spectrochim Acta A Mol Biomol Spectrosc. 2011; 79(5):1461-1465.
[44] Sheny DS, Mathew J, Philip D. Phytosynthesis of Au, Ag and $\mathrm{Au}-\mathrm{Ag}$ bimetallic nanoparticles using aqueous extract and dried leaf of Anacardium occidentale. Spectrochim Acta A Mol Biomol Spectrosc. 2011, 79(1):254-262.

[45] Philip D, Unni C, Aromal SA, Vidhu VK. Murraya Koenigii leaf-assisted rapid green synthesis of silver and gold nanoparticles. Spectrochim Acta A Mol Biomol Spectrosc. 2011; 78(2):899-904.

[46] Philip D. Rapid green synthesis of spherical gold nanoparticles using Mangifera indica leaf. Spectrochim Acta A Mol Biomol Spectrosc. 2010; 77(4):807-810. 